\title{
Analytical Method Validation of Simultaneous determination of Spironolactone and Furosemide in tablet formulation and its Statistical evaluation
}

\author{
Rajesh R. Ram ${ }^{1, *}$, Vijay R. Ram ${ }^{2}$, Hitendra S. Joshi ${ }^{3}$ \\ ${ }^{1}$ Department of Mathamatics, Bahauddin Science College, Junagadh, Gujarat, India \\ ${ }^{2}$ Department of Chemistry, KSKV Kachchh University, Bhuj, Gujarat, India \\ ${ }^{3}$ Department of Chemistry, Saurashtra University, Rajkot, Gujarat, India \\ *E-mail address: ram_vijay1982@yahoo.co.in
}

\begin{abstract}
The objective of current study was to Statistical Comparison for Precision and Intermediate Precision study for Analytical Method Validation of Spironolactone and Furosemide in tablet formulation and developed easy, exact and correct isocratic stability indicating reversed phase HPLC assay method and validated for determination of Spironolactone and Furosemide in solid pharmaceutical dosage forms. Isocratic RP-HPLC separation was achieved on an SGE make $150^{\times}$ 4.6mm SS Wakosil II 5C18RS $5 \mu \mathrm{m}$ column (Part Number: 206610 and Serial Number: A01-063) using mobile phase of Acetonitrile- Ammonium acetate buffer $(50: 50, \mathrm{v} / \mathrm{v})$ at a flow rate of $1.1 \mathrm{ml} / \mathrm{min}$ and the detection was carried out at $254 \mathrm{~nm}$ using photo-diode array detector. The method was validated for specificity, linearity, precision, accuracy, robustness and solution stability. The method was linear in the drug concentration range of $40-160 \mu \mathrm{g} / \mathrm{ml}$ with a correlation coefficient 0.9977 and 0.9953 for Spironolactone and Furosemide respectively. The precision (RSD) amongst six-sample preparation was $0.87 \%$ and $1.1 \%$ for Spironolactone and Furosemide respectively. For repeatability and intermediate precision (RSD) amongst six-sample preparation was $0.46 \%$ and $0.20 \%$ for Spironolactone and Furosemide respectively. As result shown that for furosemide, \% RSD was $1.12 \%$ and in ANOVA study Significance $F$ value found 0.625502408 and for spironolactone Precision study and Intermediate precision study \% RSD was 0.68 , in ANOVA study Significance F value found 0.905843808 .
\end{abstract}

Keywords: Statistical Comparison; Precision and Intermediate Precision study; Analytical Method Validation; Spironolactone; Furosemide; Tablet Formulation

\section{INTRODUCTION}

Stress testing is a part of developmental strategy under the ICH requirements and is carried out under more severe conditions than accelerated conditions. These studies serve to give information on drug's inherent stability and help in the validation of analytical methods to be used in stability studies [1-3]. It is suggested that stress testing should include the effect of temperature, light, oxidizing agents as well as susceptibility across a wide range of $\mathrm{pH}$ values. It is also recommended that analysis of stability sample should de done through the use of a validated stability testing methods. 


\section{1. Introduction of drug}

\section{1. 1. Introduction of furosemide}

Furosemide is chemically 4-Chloro-2-(furan-2-ylmethylamino)-5-sulfa moylbenzoic acid (Figure 1). Its CAS number is 54-31-9. Its molecular formula is $\mathrm{C}_{12} \mathrm{H}_{11} \mathrm{ClN}_{2} \mathrm{O}_{5} \mathrm{~S}$ having molecular weight $330.74 \mathrm{gm} / \mathrm{mole}$. Furosemide, an anthranilic acid derivative, is a potent diuretic that inhibits the active reabsorption of chloride in the diluting segment of the loop of Henle, thus preventing the reabsorption of sodium, which passively follows chloride [4]. This loop diuretic is commonly used for the treatment of renal diseases, congestive heart failure and hypertension [5].<smiles>NS(=O)(=O)c1cc(C(=O)O)c(NCc2ccco2)cc1Cl</smiles>

Figure 1. 4-Chloro-2-(furan-2-ylmethylamino)-5-sulfamoylbenzoic acid.

\section{1. 2. Introduction of spironolactone}

Spironolactone is chemically $7 \alpha$-Acetylthio-3-oxo-17 $\alpha$-pregn-4-ene-21,17-carbolactone (Figure 2). Its molecular formula is $\mathrm{C}_{24} \mathrm{H}_{32} \mathrm{O}_{4} \mathrm{~S}$ having molecular weight $416.58 \mathrm{gm} / \mathrm{mole}$. Spironolactone inhibits the effect of aldosterone by competing for intracellular aldosterone receptors in the distal tubule cells (it actually works on aldosterone receptors in the collecting duct). This increases the excretion of water and sodium, while decreasing the excretion of potassium. Spironolactone has a fairly slow onset of action, taking several days to develop, and similarly the effect diminishes slowly. Spironolactone has anti-androgen activity by binding to the androgen receptor and preventing it from interacting with dihydro testosterone [6]. Various publications are available regarding determination method of Spironolactone and Furosemide but most of the methods are applicable to alone Spironolactone or Furosemide in pharmaceutical dosage form or in biological fluids. Potentiometric [7], colorimetric estimation [8], thin-layer chromatography [9], fluorometrically [10], complexation [11], flow injection chemiluminescence method [12], proton nuclear magnetic resonance spectroscopic [13] and HPLC methods [14] are reported. Only four methods are reported for the simultaneous determination of spironolactone and furosemide. One method is reported for HPLC analysis. [15] As far as our knowledge is concern, no method for the determination of spironolactone and furosemide in combine dosage forms has been published. The previous published methods are not directly applicable for this issue and need more investigation for method development and validation. 


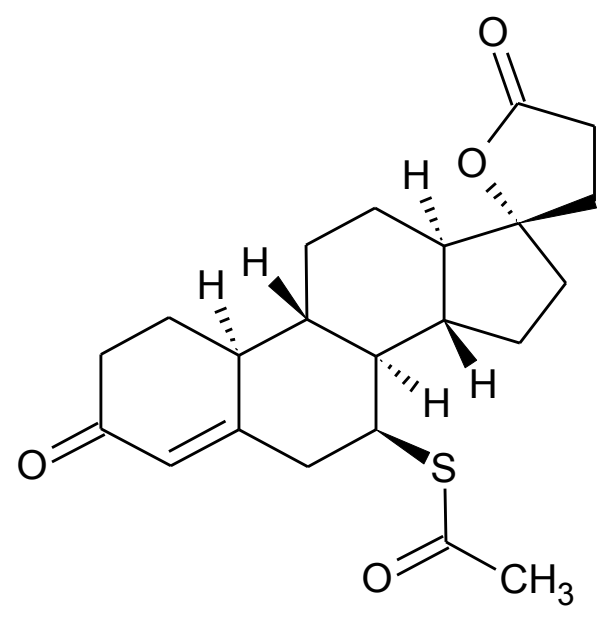

Figure 2. 7 $\alpha$-Acetylthio-3-oxo-17 $\alpha$-pregn-4-ene-21,17-carbolactone.

\section{EXPERIMENTAL}

\section{1. Materials}

Spironolactone and Furosemide standard of was provided by Alembic Pharmaceuticals Ltd., Baroda (India). Spironolactone and Furosemide tablets containing $50 \mathrm{mg}$ Spironolactone and $20 \mathrm{mg}$ Furosemide and the inactive ingredient used in drug matrix were obtained from market. HPLC grade acetonitrile and water were obtained from Spectrochem Pvt. Ltd., Mumbai (India). Analytical grade ammonium acetate, hydrochloric acid, glacial acetic acid, sodium hydroxide pellets and 30\% v/v hydrogen peroxide solution were obtained from Ranbaxy Fine Chemicals, New Delhi (India).

\section{2. Instrumentation}

The chromatographic system used to perform development and validation of this assay method was comprised of a LC-10ATvp binary pump, a SPD-M10Avp photo-diode array detector and a rheodyne manual injector model $7725 \mathrm{i}$ with $20 \mu 1$ loop (Shimadzu, Kyoto, Japan) connected to a multi-instrument data acquisition and data processing system (Class-VP 6.13 SP2, Shimadzu).

\section{3. Chromatographic conditions}

Chromatographic analysis was performed on a SGE make SS Wakosil II 5C18RS column $(150 \mathrm{~mm} \times 4.6 \mathrm{~mm}$ i.d., $5 \mu \mathrm{m}$ particle size) column. The mobile phase consisted of acetonitrile $-0.01 \mathrm{M}$ ammonium acetate buffer $\mathrm{pH} 3.9(50: 50, \mathrm{v} / \mathrm{v})$. To prepare the buffer solution, $0.7708 \mathrm{~g}$ ammonium acetate were weighed and dissolve in $1000 \mathrm{ml}$ HPLC grade water and then adjusted to $\mathrm{pH} 3.9$ with glacial acetic acid. Mobile phase was filtered through a $0.45 \mu \mathrm{m}$ nylon membrane (Millipore Pvt. Ltd. Bangalore, India) and degassed in an ultrasonic bath (Spincotech Pvt. Ltd., Mumbai). The flow rate of the mobile phase was adjusted to $1.1 \mathrm{ml} / \mathrm{min}$ and the injection volume was $20 \mu \mathrm{l}$. Detection was performed at $254 \mathrm{~nm}$. 


\section{4. Standard preparation}

Spironolactone standard stock solution containing $500 \mu \mathrm{g} / \mathrm{ml}$ was prepared in a $100 \mathrm{ml}$ volumetric flask by dissolving $50.00 \mathrm{mg}$ of Spironolactone and then diluted to volume with diluent. Further take $10 \mathrm{ml}$ of this stock solution in $50 \mathrm{ml}$ volumetric flask and make up to mark with diluent (this standard solution of $100 \mu \mathrm{g} / \mathrm{ml}$ ). And for a Furosemide standard stock solution containing $200 \mu \mathrm{g} / \mathrm{ml}$ was prepared in a $100 \mathrm{ml}$ volumetric flask by dissolving 20.00 $\mathrm{mg}$ of Furosemide and then diluted to volume with diluent. Further take $10 \mathrm{ml}$ of this stock solution in $50 \mathrm{ml}$ volumetric flask and make up to mark with diluent (this standard solution of $40 \mu \mathrm{g} / \mathrm{ml})$.

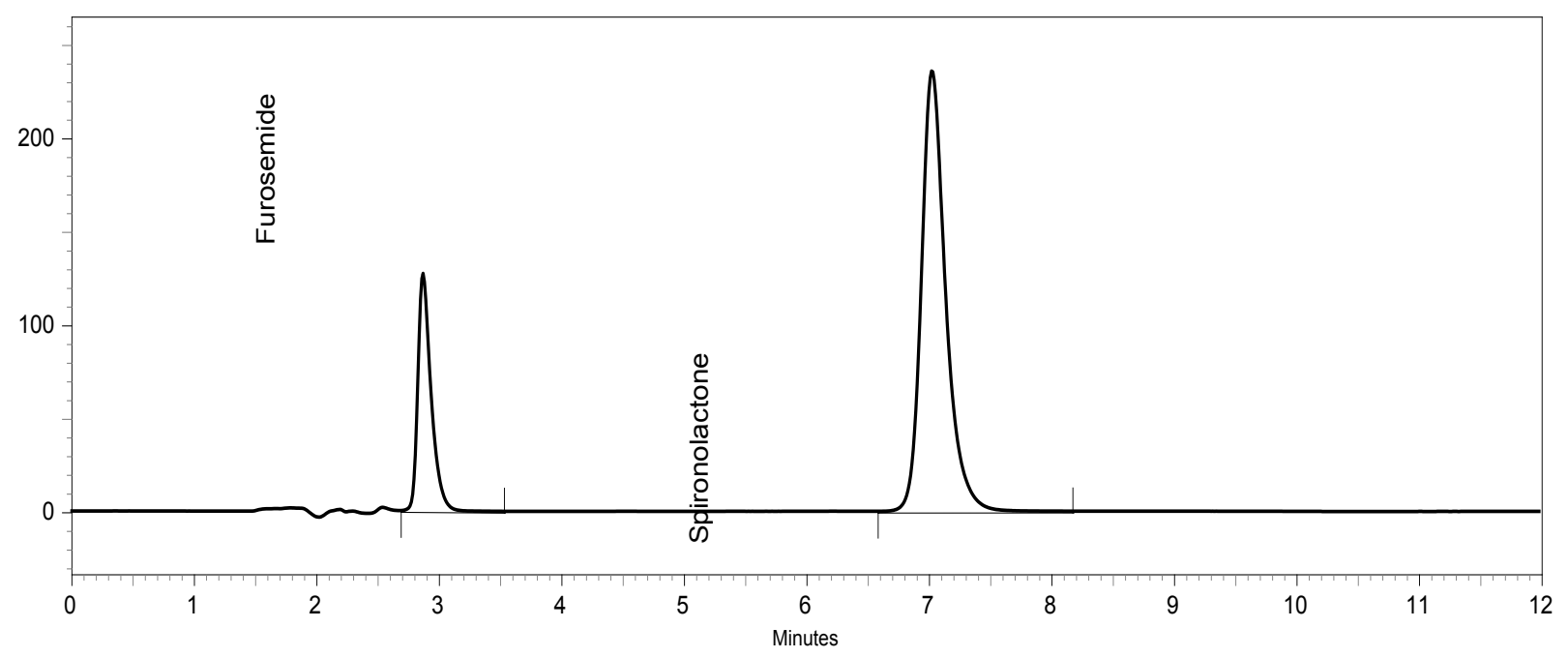

Figure 3. Chromatogram of standard preparation.

\subsection{Test preparation}

Twenty tablets were weighed and the average weight of tablet was determined. From these, five tablets were weighed and transfer into a $500 \mathrm{ml}$ volumetric flask. About $50 \mathrm{ml}$ of diluent was added and sonicated for a minimum $30 \mathrm{~min}$. with intermittent shaking. Then content was brought back to room temperature and diluted to volume with diluent. The sample was filtered through $0.45 \mu \mathrm{m}$ nylon syringe filter. Further take $10 \mathrm{ml}$ of this stock solution in $50 \mathrm{ml}$ of volumetric flask and make up to mark with diluent. The concentration obtained was $100 \mu \mathrm{g} / \mathrm{ml}$ of Spironolactone and $40 \mu \mathrm{g} / \mathrm{ml}$ of Furosemide.

\section{RESULT AND DISCUSSION}

\section{1. Method validation}

\section{1. Specificity study}

The evaluation of the specificity of the method was determined against placebo. The interference of the excipients of the claimed placebo present in pharmaceutical dosage form was derived from placebo solution. Further the specificity of the method toward the drug was established by means of checking the interference of the degradation products in the drug quantification for assay during the forced degradation study. 
Table 1. Precision study for Analytical Method Validation of Furosemide Spironolactone.

\begin{tabular}{|c|c|c|c|c|c|c|c|}
\hline \multicolumn{4}{|c|}{ Furosemide } & \multicolumn{4}{|c|}{ Spironolactone } \\
\hline Description & $\begin{array}{l}\text { Mean } \\
\text { area }\end{array}$ & Wt. (mg) & $\begin{array}{c}\% \\
\text { Assay }\end{array}$ & Description & $\begin{array}{c}\text { Mean } \\
\text { area }\end{array}$ & $\begin{array}{l}\text { Wt. } \\
(\mathrm{mg})\end{array}$ & $\begin{array}{c}\% \\
\text { Assay }\end{array}$ \\
\hline Set 1 & 998819 & 1071.5 & 101.0 & Set 1 & 3366197 & 1071.5 & 101.0 \\
\hline Set 2 & 999963 & 1070.5 & 101.1 & Set 2 & 3379481 & 1070.5 & 101.4 \\
\hline Set 3 & 978857 & 1071.3 & 98.9 & Set 3 & 3311436 & 1071.3 & 99.4 \\
\hline Set 4 & 975578 & 1070.3 & 98.6 & Set 4 & 3366585 & 1070.4 & 101.0 \\
\hline Set 5 & 987644 & 1071.4 & 99.8 & Set 5 & 3351654 & 1071.4 & 100.6 \\
\hline \multirow[t]{4}{*}{ Set 6} & 1003532 & 1072 & 101.4 & Set 6 & 3397057 & 1072 & 101.9 \\
\hline & & Mean & 100.1 & & & Mean & 100.9 \\
\hline & & Stdev & 1.19 & & & Stdev & 0.87 \\
\hline & & $\%$ RSD & 1.19 & & & $\begin{array}{c}\% \\
\text { RSD }\end{array}$ & 0.87 \\
\hline $\begin{array}{l}\text { Standard } \\
\text { mean area }\end{array}$ & 995588 & $\begin{array}{c}\text { Standard } \\
\text { mean } \\
\text { area }\end{array}$ & & $\begin{array}{c}\text { Standard mean } \\
\text { area }\end{array}$ & 3374356 & & \\
\hline $\begin{array}{l}\text { Standard } \\
\text { wt. (mg) }\end{array}$ & 19.8 & $\begin{array}{l}\text { Standard } \\
\text { wt. (mg) }\end{array}$ & & $\begin{array}{l}\text { Standard wt. } \\
\text { (mg) }\end{array}$ & 49.8 & & \\
\hline Test wt & 1071.3 & Test wt & & Test wt & 1071.3 & & \\
\hline $\begin{array}{l}\text { Label claim } \\
\text { (mg) }\end{array}$ & 20 & $\begin{array}{l}\text { Label } \\
\text { claim } \\
(\mathrm{mg})\end{array}$ & & $\begin{array}{l}\text { Label claim } \\
\qquad(\mathrm{mg})\end{array}$ & 50 & & \\
\hline $\begin{array}{l}\text { Average } \\
\text { Wt. (mg) }\end{array}$ & 217.8 & $\begin{array}{l}\text { Average } \\
\text { Wt. (mg) }\end{array}$ & & $\begin{array}{l}\text { Average Wt. } \\
(\mathrm{mg})\end{array}$ & 217.8 & & \\
\hline
\end{tabular}


Table 2. Intermediate Precision study for Analytical Method Validation of Furosemide Spironolactone.

\begin{tabular}{|c|c|c|c|c|c|c|c|}
\hline \multicolumn{4}{|c|}{ Furosemide } & \multicolumn{4}{|c|}{ Spironolactone } \\
\hline Description & $\begin{array}{l}\text { Mean } \\
\text { area }\end{array}$ & Wt. (mg) & $\begin{array}{c}\% \\
\text { Assay }\end{array}$ & Description & $\begin{array}{c}\text { Mean } \\
\text { area }\end{array}$ & $\begin{array}{l}\text { Wt. } \\
(\mathrm{mg})\end{array}$ & $\begin{array}{c}\% \\
\text { Assay }\end{array}$ \\
\hline Set 1 & 1003949 & 1071.5 & 98.9 & Set 1 & 3496814 & 1071.5 & 100.7 \\
\hline Set 2 & 1004029 & 1070.5 & 98.9 & Set 2 & 3500878 & 1070.5 & 100.9 \\
\hline Set 3 & 1001844 & 1071.3 & 98.7 & Set 3 & 3480761 & 1071.3 & 100.3 \\
\hline Set 4 & 1000111 & 1070.3 & 98.5 & Set 4 & 3459055 & 1070.3 & 99.7 \\
\hline Set 5 & 1000388 & 1071.4 & 98.5 & Set 5 & 3497922 & 1071.4 & 100.8 \\
\hline \multirow[t]{4}{*}{ Set 6} & 999289 & 1072 & 98.4 & Set 6 & 3480035 & 1072 & 100.3 \\
\hline & & Mean & 98.6 & & & Mean & 100.4 \\
\hline & & Stdev & 0.20 & & & Stdev & 0.46 \\
\hline & & $\%$ RSD & 0.20 & & & $\begin{array}{c}\% \\
\mathrm{RSD}\end{array}$ & 0.46 \\
\hline $\begin{array}{l}\text { Standard } \\
\text { mean area }\end{array}$ & 1052789 & & & $\begin{array}{c}\text { Standard mean } \\
\text { area }\end{array}$ & 3577951 & & \\
\hline $\begin{array}{l}\text { Standard } \\
\text { wt. (mg) }\end{array}$ & 20.4 & & & $\begin{array}{l}\text { Standard wt. } \\
(\mathrm{mg})\end{array}$ & 50.7 & & \\
\hline $\begin{array}{l}\text { Test wt } \\
(\mathrm{mg})\end{array}$ & 1071.3 & & & Test wt (mg) & 1071.3 & & \\
\hline $\begin{array}{l}\text { Label claim } \\
\text { (mg) }\end{array}$ & 20 & & & $\begin{array}{l}\text { Label claim } \\
\text { (mg) }\end{array}$ & 50 & & \\
\hline $\begin{array}{l}\text { Average } \\
\text { Wt. (mg) }\end{array}$ & 217.8 & & & $\begin{array}{l}\text { Average Wt. } \\
(\mathrm{mg})\end{array}$ & 217.8 & & \\
\hline
\end{tabular}


Table 3. Statistical Comparison of Precision and Intermediate Precision study for Analytical Method Validation of Furosemide in tablet formulation.

\begin{tabular}{|c|c|c|c|c|c|c|}
\hline \multirow{7}{*}{$\begin{array}{l}\text { Precision } \\
\text { study }\end{array}$} & \multicolumn{6}{|c|}{ FUROSEMIDE } \\
\hline & Set 1 & 101.0 & \multicolumn{2}{|c|}{ For Precision } & \multicolumn{2}{|c|}{ For Intermediate Precision } \\
\hline & Set 2 & 101.1 & Mean & 101.35 & Mean & 100.3333 \\
\hline & Set 3 & 98.9 & $\begin{array}{l}\text { Standard } \\
\text { Error }\end{array}$ & 0.287228132 & $\begin{array}{l}\text { Standard } \\
\text { Error }\end{array}$ & 0.567255 \\
\hline & Set 4 & 98.6 & Median & 101.15 & Median & 99.8 \\
\hline & Set 5 & 99.8 & Mode & $\# \mathrm{~N} / \mathrm{A}$ & Mode & $\# \mathrm{~N} / \mathrm{A}$ \\
\hline & Set 6 & 101.3 & $\begin{array}{l}\text { Standard } \\
\text { Deviation }\end{array}$ & 0.703562364 & $\begin{array}{l}\text { Standard } \\
\text { Deviation }\end{array}$ & 1.389484 \\
\hline \multirow{10}{*}{$\begin{array}{l}\text { Intermediate } \\
\text { precision } \\
\text { study }\end{array}$} & Set 1 & 98.9 & $\begin{array}{c}\text { Sample } \\
\text { Variance }\end{array}$ & 0.495 & $\begin{array}{c}\text { Sample } \\
\text { Variance }\end{array}$ & 1.930667 \\
\hline & Set 2 & 98.9 & Kurtosis & $\begin{array}{c}- \\
1.398102234\end{array}$ & Kurtosis & -1.40619 \\
\hline & Set 3 & 98.7 & Skewness & 0.478086455 & Skewness & 0.698096 \\
\hline & Set 4 & 98.5 & Range & 1.8 & Range & 3.5 \\
\hline & Set 5 & 98.5 & Minimum & 100.5 & Minimum & 98.8 \\
\hline & Set 6 & 98.4 & Maximum & 102.3 & Maximum & 102.3 \\
\hline & Mean & 99.4 & Sum & 608.1 & Sum & 602 \\
\hline & Stdev & 1.12 & Count & 6 & Count & 6 \\
\hline & $\%$ RSD & 1.12 & $\begin{array}{c}\text { Confidence } \\
\text { Level }(95.0 \%)\end{array}$ & 0.738342213 & $\begin{array}{c}\text { Confidence } \\
\text { Level }(95.0 \%)\end{array}$ & 1.458172 \\
\hline & $\begin{array}{c}\text { FUROSEMIDE } \\
\text { SUMMARY } \\
\text { OUTPUT } \\
\end{array}$ & & & & & \\
\hline $\begin{array}{c}\text { Regression } \\
\text { Statistics }\end{array}$ & & & & & & \\
\hline
\end{tabular}




\begin{tabular}{|c|c|c|c|c|c|c|}
\hline Multiple R & 0.255205564 & & & & & \\
\hline R Square & 0.06512988 & & & & & \\
\hline $\begin{array}{l}\text { Adjusted R } \\
\text { Square }\end{array}$ & -0.16858765 & & & & & \\
\hline $\begin{array}{l}\text { Standard } \\
\text { Error }\end{array}$ & 0.234357888 & & & & & \\
\hline Observations & 6 & & & & & \\
\hline \multirow[t]{2}{*}{ ANOVA } & & & & & & \\
\hline & $d f$ & $S S$ & $M S$ & $F$ & $\begin{array}{c}\text { Significance } \\
F\end{array}$ & \\
\hline Regression & 1 & 0.015305522 & 0.015305522 & 0.278669 & 0.625502408 & \\
\hline Residual & 4 & 0.219694478 & 0.05492362 & & & \\
\hline \multirow[t]{2}{*}{ Total } & 5 & 0.235 & & & & \\
\hline & Coefficients & $\begin{array}{l}\text { Standard } \\
\text { Error }\end{array}$ & $t$ Stat & P-value & Lower 95\% & Upper 95\% \\
\hline Intercept & 93.99657614 & 8.815636944 & 10.66248267 & 0.000438 & 69.5204441 & 118.4727082 \\
\hline X Variable 1 & 0.046734418 & 0.088530383 & 0.527891288 & 0.625502 & -0.19906533 & 0.292534165 \\
\hline
\end{tabular}

\section{1. 2. Linearity}

Linearity test solutions for the assay method were prepared at seven concentration levels from 40 to $160 \%$ of assay analyte concentration $(40,60,80,100,120,140$ and $160 \mu \mathrm{g} / \mathrm{ml})$. The peak areas versus concentration data were evaluated by linear regression analysis.

\section{1. 3. Precision}

The precision of the assay method was evaluated in terms of repeatability by carrying out six independent assays of Spironolactone and Furosemide test sample preparation and 
calculated the \% RSD of assay (intraday). Intermediate precision of the method was checked by performing same procedure on the different day (interday) by another person under the same experimental condition.

\section{1. 4. STATISTICAL EVALUATION}

Statistical evaluation is given in table no. 1, 2, 3 and 4. And Formula for assay calculation was

$\%$ Assay $=$ Average Test area / Average std. area * Std. wt. * 405.48/441.94 / 250*250/ Test wt. / Label claim * Average wt. * Potency of std.

Table 4. Statistical Comparison of Precision and Intermediate Precision study for Analytical Method Validation of Spironolactone in tablet formulation.

\begin{tabular}{|c|c|c|c|c|c|c|}
\hline \multirow{7}{*}{$\begin{array}{c} \\
\text { Precision } \\
\text { study }\end{array}$} & \multicolumn{6}{|c|}{ SPIRONOLACTONE } \\
\hline & \multirow{2}{*}{$\begin{array}{l}\text { Set } 1 \\
\text { Set } 2\end{array}$} & \multirow{2}{*}{$\begin{array}{r}101.0 \\
101.4\end{array}$} & \multicolumn{2}{|c|}{ For Precision } & \multicolumn{2}{|c|}{ For Intermediate Precision } \\
\hline & & & Mean & 101.35 & Mean & 100.3333 \\
\hline & Set 3 & 99.4 & $\begin{array}{c}\text { Standard } \\
\text { Error }\end{array}$ & 0.287228132 & $\begin{array}{l}\text { Standard } \\
\text { Error }\end{array}$ & 0.567255 \\
\hline & Set 4 & 101.0 & Median & 101.15 & Median & 99.8 \\
\hline & Set 5 & 100.6 & Mode & $\# \mathrm{~N} / \mathrm{A}$ & Mode & $\# \mathrm{~N} / \mathrm{A}$ \\
\hline & Set 6 & 101.9 & $\begin{array}{l}\text { Standard } \\
\text { Deviation }\end{array}$ & 0.703562364 & $\begin{array}{c}\text { Standard } \\
\text { Deviation }\end{array}$ & 1.389484 \\
\hline \multirow{6}{*}{$\begin{array}{c}\text { Intermediate } \\
\text { precision } \\
\text { study }\end{array}$} & Set 1 & 100.7 & $\begin{array}{c}\text { Sample } \\
\text { Variance }\end{array}$ & 0.495 & $\begin{array}{c}\text { Sample } \\
\text { Variance }\end{array}$ & 1.930667 \\
\hline & Set 2 & 100.9 & Kurtosis & $\begin{array}{c}- \\
1.398102234\end{array}$ & Kurtosis & -1.40619 \\
\hline & Set 3 & 100.3 & Skewness & 0.478086455 & Skewness & 0.698096 \\
\hline & Set 4 & 99.7 & Range & 1.8 & Range & 3.5 \\
\hline & Set 5 & 100.8 & Minimum & 100.5 & Minimum & 98.8 \\
\hline & Set 6 & 100.3 & Maximum & 102.3 & Maximum & 102.3 \\
\hline & Mean & 100.7 & Sum & 608.1 & Sum & 602 \\
\hline & Stdev & 0.69 & Count & 6 & Count & 6 \\
\hline
\end{tabular}




\begin{tabular}{|c|c|c|c|c|c|c|}
\hline & $\%$ RSD & 0.68 & $\begin{array}{c}\text { Confidence } \\
\text { Level(95.0\%) }\end{array}$ & 0.738342213 & $\begin{array}{c}\text { Confidence } \\
\text { Level }(95.0 \%)\end{array}$ & 1.458172 \\
\hline & $\begin{array}{c}\text { FUROSEMIDE } \\
\text { SUMMARY } \\
\text { OUTPUT }\end{array}$ & & & & & \\
\hline \multicolumn{7}{|l|}{$\begin{array}{l}\text { Regression } \\
\text { Statistics }\end{array}$} \\
\hline Multiple R & 0.062853564 & & & & & \\
\hline R Square & 0.00395057 & & & & & \\
\hline $\begin{array}{l}\text { Adjusted R } \\
\text { Square }\end{array}$ & -0.245061787 & & & & & \\
\hline $\begin{array}{l}\text { Standard } \\
\text { Error }\end{array}$ & 0.497762288 & & & & & \\
\hline Observations & 6 & & & & & \\
\hline \multicolumn{7}{|l|}{ ANOVA } \\
\hline & $d f$ & $S S$ & $M S$ & $F$ & $\begin{array}{c}\text { Significance } \\
F\end{array}$ & \\
\hline Regression & 1 & 0.003930818 & 0.003930818 & 0.015865 & 0.905843808 & \\
\hline Residual & 4 & 0.991069182 & 0.247767296 & & & \\
\hline \multirow[t]{2}{*}{ Total } & 5 & 0.995 & & & & \\
\hline & Coefficients & $\begin{array}{l}\text { Standard } \\
\text { Error }\end{array}$ & t Stat & P-value & Lower 95\% & Upper 95\% \\
\hline Intercept & 97.28805031 & 25.10439313 & 3.875339659 & 0.017912 & 27.58708089 & 166.9890197 \\
\hline X Variable 1 & 0.031446541 & 0.249662563 & 0.125956173 & 0.905844 & $\begin{array}{c}- \\
0.661727859\end{array}$ & 0.72462094 \\
\hline
\end{tabular}

\section{CONCLUSION}

In this current study a new analytical method has been developed to be routinely applied to simultaneous determine Spironolactone and Furosemide in pharmaceutical dosage form. The developed procedure has been statistically evaluated for Precision study and Intermediate precision study. And as result shown that for furosemide, $\%$ RSD was $1.12 \%$ and in ANOVA study Significance $F$ value found 0.625502408 and for spironolactone Precision study and Intermediate precision study \% RSD was 0.68 , in ANOVA study Significance $F$ value found 
0.905843808. So, it is concluded that current method passes through its Significance value. Hence, the method is recommended for routine quality control analysis and also stability sample analysis.

\section{Acknowledgements}

The authors are thankful to Department of Chemistry, Saurashtra University, Rajkot (INDIA) and Department of Chemistry, KSKV Kachchh University for providing analytical facilities.

\section{Reference}

[1] S. Singh, M. Bakshi, Pharm. Technol. 24 (2004) 1.

[2] M. Bakshi, B. Singh, A. Singh, S. Singh, J. Pharm. Biomed. Anal. 26 (2001) 1011.

[3] M. Bakshi, S. Singh, J. Pharm. Biomed. Anal. 28 (2002) 891.

[4] E. Berardesca, P. Gabba, G. Ucci, G. Borroni, G. Rabbiosi, Int. J. Tissue React. 10(2) (1988) 115.

[5] H. R. Jacobson, J. P. Kokko, Annu. Rev. Pharmacol. Toxicol. 16 (1976) 201.

[6] www.sanofi-aventis.com

[7] www.archneur.ama-assn.org

[8] Felipe Silva Semaan, Paulo Alberto Nogueira, Eder Tadeu Gomes Cavalheiro, Analytical Letters 41(1) (2008) 66-79.

[9] Juan Xie, Fang Yang, Yu Zhai, Jie Yao, Weiqun Zhang, Zhongguo Yaofang. Tetrahedron 18(31) (2007) 2454-2455.

[10] Pradeep Mishra, D. Katrolia, R. K. Agrawal, Current Science 58(9) (1989) 503-505.

[11] Lilo O. Guerello, Jose Dobrecky, Revista Farmaceutica 111(1-2) (1969) 13-16.

[12] B Wesley-Hadzija, A M Mattocks, Journal of chromatography 229(2) (1982) 425-32.

[13] A. Goelcue, Journal of Analytical Chemistry 61(8) (2006) 748-754.

[14] dward F. Salim, A. Haussler, J. B. Vaughan, Journal of Pharmaceutical Sciences 57(4) (1968) 640-641.

[15] V. Ram, P. Dave and H. Joshi, Journal of Chromatographic Science 50(8) (2012) 721726. 